\title{
Experiments with Balancing on Irregular Terrains using the Dreamer Mobile Humanoid Robot
}

\author{
Luis Sentis, University of Texas at Austin, USA \\ Josh Petersen, University of Texas at Austin, US \\ Roland Philippsen, Halmstad University, Sweden
}

\begin{abstract}
We investigate controllers for mobile humanoid robots that maneuver in irregular terrains while performing accurate physical interactions with the environment and with human operators and test them on Dreamer, our new robot with a humanoid upper body (torso, arm, head) and a holonomic mobile base (triangularly arranged Omni wheels). All its actuators are torque controlled, and the upper body provides redundant degrees of freedom. We developed new dynamical models and created controllers that stabilize the robot in the presence of slope variations, while it compliantly interacts with humans.

This paper considers underactuated free-body dynamics with contact constraints between the wheels and the terrain. Moreover, Dreamer incorporates a biarticular mechanical transmission that we model as a force constraint. Using these tools, we develop new compliant multiobjective skills and include selfmotion stabilization for the highly redundant robot.
\end{abstract}

\section{INTRODUCTION}

Real-world terrains exhibit curvature changes, discrete slabs, granular debris, etc. Any robot that is supposed to help automate industrial and urban related chores, in the presence of irregular terrains and human operators, has to be able to maneuver, manipulate and interact safely, accurately and robustly in these type of environments. And it will have to do so while safely and physically interacting with human users.

This paper contributes a theoretical and experimental study of a control approach with such capabilities. It is based on the whole-body control framework, which provides a powerful methodology to incorporate constrains and build robot skills as hierarchies of motion and force tasks. We formulate unified dynamics for tasks and constraints and use them to construct compliant controllers for our humanoid torque-controlled mobile robot Dreamer. We describe, model, and control two fundamental constraints, prove the stability of our method for controlling motion redundancy, and show how our control approach can naturally implement kinesthetic interaction with the robot. The experiments provide an empirical proof of concept for the developed concepts and methodology.

The most fundamental constraint for any mobile platform is the contact between its wheels and the ground. It is common to build mobile bases that can be treated as planar joints, but this requires them to be heavy and large to create passive righting moments that exceed any expected tipping moment. This requirement severely limits areas for potential deployment, which is why we designed Dreamer with a smaller and more lightweight base, and chose to address stability using an active approach inspired by our earlier work on legged humanoids. By incorporating the full mobility of the base,

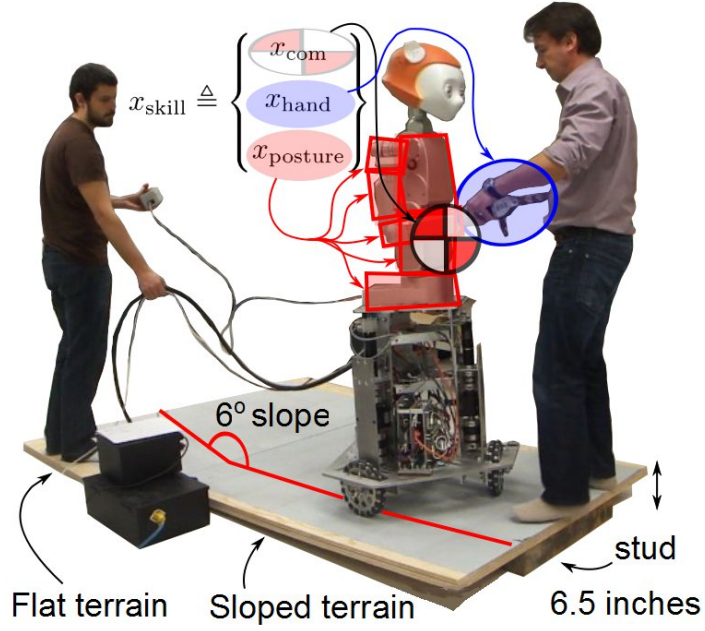

Fig. 1. Experimental setup with an indication of the coordinates used by the physial intraction skill described in the experimental section.

as opposed to the frequently unstated planarity assumption, it becomes possible to estimate balance coordinates due to variations in slope, account for free-body dynamic effects such as conservation of momenta, and create an infrastructure that is dynamically correct in 3D space. For Dreamer, there is another fundamental constraint due to the mechanical coupling of two motion axes in its torso. This biarticular joint provides a large range of motion while keeping the mechatronics very compact.

Stable control of redundancies is a general issue for manipulators with more degrees of freedom (DOF) than required for fulfilling a particular skill. In this paper, we contribute a detailed stability analysis of our method of addressing such redundancies.

\section{A. Related Work}

Our line of research is a direct successor of [11]. Here, a mobile omnidirectional manipulator for flat terrain was developed using caster wheels and a Puma industrial manipulator with an Operational Control layer [14]. Our work is a departure from the previous one in various respects: (1) we implement wholebody dynamics that incorporate the free floating underactuated effects, (2) we describe the dependencies between wheels as contact and rolling constraints, (3) we implement a dynamically correct prioritized control strategy, and (4) we control balance to prevent falling in the rough terrains. As such, we provide a platform that is more capable to adapt to outdoor 
environments and is more dexterous in executing whole-body skills.

Although, whole-body compliant control in mobile robots and humanoids has been thoroughly studied by us [15], [21] and by our predecesors [3], [1] more recent implementations include [17], [9], [6]. In these works whole-body torque control of the base and the upper body are considered. In particular, in [9], [6] the coordination of whole-body torques is accomplished by separating upper-body torques and an admittance control strategy for the velocity controlled base. Limitations of these works include flat terrain assumptions, lacking balancing capabilities and considering static contact conditions on the base wheels.

Balancing is an important component of our approach to handling rough terrains. In [8], a large wheeled robot with reconfigurable pose is used to maneuver in outdoor backterrains. Compared to our work, the previous one does not utilize whole-body dynamics nor contact state estamination. In turn, the robot will perform poorly when executing fast dynamic maneuvers. A simple wheeled robot that balances to operate in rough roads has been proposed in [16]. They use wheeled inverted pendulum dynamics to stabilize balance through state feedback control. This work shares balance capabilities with our approach but is limited in that it does not have manipulation capabilities.

As for the control architecture, our software relates (within a limited architectural scope) to [2], but in contrast we provide whole-body dynamic models apt for the control of mobile humanoids under contact constraints. When compared to [13] our software provides a multi-objective torque control layer that enables to utilize the robot's motion and contact redundancy more efficiently.

\section{B. The Dreamer Humanoid Robot Hardware}

The main hardware tool that we use for this study is a new mobile dexterous humanoid robot. It includes a torso, an arm, a hand, an anthropomorphic head developed by Meka Robotics, and a torque-controlled holonomic base developed by our lab. The actuators for the base and the upper body, except for the head, contain torque/force sensors that enable Elmo amplifiers to implement current or torque feedback. An Ethercat serial bus communicates with sensors and motor amplifiers from a single computer system. A PC running Ubuntu Linux with the RTAI Realtime Kernel runs the models and control infrastructure described in this project. The holonomic base contains rotary torque sensors as well as the inertial measurement unit (IMU) 3DM-GX3-25 from MicroStrain. It achieves holonomic motion and force capabilities by utilizing Omni wheels located in a equilateral triangular configuration.

\section{Constrained, UnderactuAted DynAmics}

The model of Dreamer's dynamic behavior is formulated using Lagrangian rigid multibody dynamics. A diagram of the modeled entities is shown in Figure 2. We start by introducing the generalized coordinate vector

$$
q \triangleq\left(q_{\text {freebody }} \quad q_{\text {wheels }} \quad q_{\text {torso }} \quad q_{\text {arm }}\right) \in \mathbb{R}^{n_{\text {dofs }}},
$$

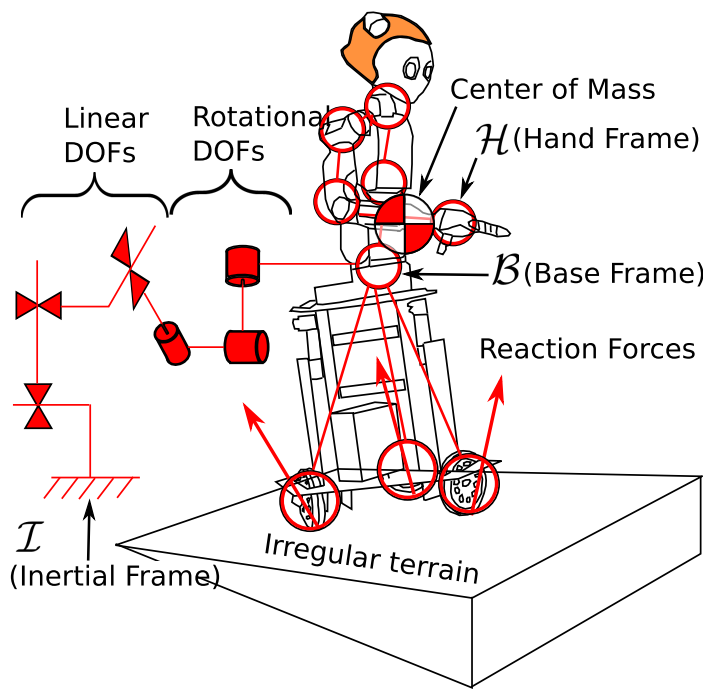

Fig. 2. "Free-body" degrees of freedom, reaction forces, and robot's kinematic chain: in order to adapt to irregular terrains, we model the robot kinematics and dynamics with respect to an inertial reference frame. This step entails adding 6 kinematic degrees of freedom of free-body movement to the kinematic description of the robot. Furthermore, rolling and contact constraints on the wheels are modeled for contact-based control.

where $q_{\text {freebody }} \in \mathbb{R}^{6}$ corresponds to the passive free-body linear and angular DOF of the base, $q_{\text {wheels }} \in \mathbb{R}^{3}$ correspond to the actuated base wheels, $q_{\text {torso }} \in \mathbb{R}^{3}$ correspond to the robot's upper torso with the biarticular constraint, and $q_{\text {arm }} \in$ $\mathbb{R}^{7}$ corresponds to the robot's actuated right arm. The equation of motion underlying the model is

$$
A(q) \ddot{q}+b(q, \dot{q})+g(q)+J_{\text {constr }}^{T} \lambda_{\text {constr }}=U^{T} \tau_{\text {control }},
$$

where $\{A, b, g\}$ are the inertial, Coriolis-centrifugal, and gravity terms; $J_{\text {constr }}$ is the constraint Jacobian (sections II-B and II-C); $\lambda_{\text {constr }}$ are Lagrangian multipliers; $U^{T}$ maps actuation forces into the system (section II-A); and $\tau_{\text {control }} \in$ $\mathbb{R}^{n_{\text {dofs }}-(1+6)}$ is the vector of motor torques. We assume $\{A, b, g\}$ known, solve for $\lambda_{\text {constr }}$ here, and develop the expressions for the remaining terms in the following subsections.

$\lambda_{\text {constr }}$ can be found by left-multiplying (2) by $J_{\text {constr }} A^{-1}$ and applying the time-derivative of the constraint equation $J_{\text {constr }} \dot{q}=0$ to yield

$$
\lambda_{\text {constr }}=\bar{J}_{\text {constr }}^{T}\left(U^{T} \tau_{\text {control }}-b-g\right)+\Lambda_{\text {constr }} \dot{J}_{\text {constr }} \dot{q},
$$

where $\bar{J}_{\text {constr }} \triangleq A^{-1} J_{\text {constr }}^{T} \Lambda_{\text {constr }}$ is the dynamically consistent generalized inverse of $J_{\text {constr }}$ and $\Lambda_{\text {constr }} \triangleq$ $\left(J_{\text {constr }} A^{-1} J_{\text {constr }}^{T}\right)^{-1}$ is the inertial matrix projected in the manifold of the constraints. These steps eliminate the constraint forces from (2), and we obtain the equation of motion of the constrained underactuated system as

$$
\begin{aligned}
A \ddot{q}+N_{\text {constr }}^{T}(b+g)+J_{\text {constr }}^{T} & \Lambda_{\text {constr }} \dot{J}_{\text {constr }} \dot{q} \\
& =\left(U N_{\text {constr }}\right)^{T} \tau_{\text {control }},
\end{aligned}
$$

where $N_{\text {constr }} \triangleq I-\bar{J}_{\text {constr }} J_{\text {constr }}$ is the dynamically consistent null space of the constraint Jacobian. 
In the following subsection, we develop $U$ and $J_{\text {constr. The }}$ latter collects various constraints. In this paper, it is defined as

$$
J_{\text {constr }} \triangleq\left(\begin{array}{c}
J_{\text {biart }} \\
J_{\text {contact }}
\end{array}\right) \in \mathbb{R}^{(1+6) \times n_{\text {dofs }}}
$$

\section{A. Underactuation Model}

There are two sources of underactuation on Dreamer, namely the six free-floating DOF (modeled as virtual unactuated prismatic and revolute joints), and the fact that the two Sagittal joints in the torso are driven by a single motor. We express this dependencies as $q_{\text {act }}=U q \in \mathbb{R}^{n_{\text {dofs }}-(1+6)}$ where

$$
U \triangleq\left(\begin{array}{cccccc}
\mathbf{0}_{3 \times 6} & \mathbf{1}_{3 \times 3} & \multicolumn{2}{c}{\mathbf{0}_{3 \times 3}} & \mathbf{0}_{3 \times 7} \\
\mathbf{0}_{2 \times 6} & \mathbf{0}_{2 \times 3} & 1 & 0 & 0 & \mathbf{0}_{2 \times 7} \\
\mathbf{0}_{7 \times 6} & \mathbf{0}_{7 \times 3} & \multicolumn{2}{c}{\mathbf{0}_{7 \times 3}} & \mathbf{1}_{7 \times 7}
\end{array}\right)
$$

is the $\left(n_{\text {dofs }}-(1+6)\right) \times n_{\text {dofs }}$ matrix that maps the biarticular and free-body DOF into the equation of motion.

\section{B. Biarticular Constraint}

Biarticular joints in our robot are located in between the hip and thorax segments of the torso. In particular, the torso has 3 degrees of freedom (DOF) - waist rotation, hip flexion/extension, thorax flexion/extension — but only the waist rotation and hip flexion/extension are directly actuated by independent motors. A steel wire runs between the hip and thorax DOF to provide actuation to the two Sagittal joints of the upper torso. This type of transmission constraint represents an underactuated DOF and creates tension forces across the wire. Because it creates position constraints, it is of a holonomic nature.

In particular, Dreamer's biarticular constraint can be modeled as $q_{\text {hip }}=q_{\text {thorax }}$. It follows that the instantaneous velocities of both joint are also equal $\left(\dot{q}_{\text {hip }}=\dot{q}_{\text {thorax }}\right)$.

Let us define the coordinate $\delta_{\text {biart }} \triangleq q_{\text {hip }}-q_{\text {thorax }}$. Then, a differential relation with respect to the generalized coordinates can be expressed as

$$
\dot{\delta}_{\text {biart }}=J_{\text {biart }} \dot{q}=0,
$$

where $\dot{q}$ is the vector of generalized joint velocities (6 for the free-body base, 3 for the wheels, 3 for the upper torso and 7 for the right arm), and the Jacobian of the biarticular constraint is expressed as

$$
J_{\text {biart }} \triangleq\left(\begin{array}{llllll}
\mathbf{0}_{1 \times 6} & \mathbf{0}_{1 \times 3} & 0 & 1 & -1 & \mathbf{0}_{1 \times 7}
\end{array}\right) \in \mathbb{R}^{1 \times n_{\text {dofs }}}
$$

\section{Rolling and Contact Constraints}

The relative velocity of the ground contact point of the $i$-th wheel, $v_{\text {contact }[i]}$, depends on the velocity of the wheel center, the angular velocity of the wheel, and the relative position of the contact point:

$$
v_{\text {contact }[i]}=v_{\text {wheel }[i]}+\omega_{\text {wheel }[i]} \times \delta_{\text {contact }[i]} \in \mathbb{R}^{3}
$$

where $v_{\text {wheel }[i]}$ and $\omega_{\text {wheel }[i]}$ are linear and angular velocities of the wheel, and $\delta_{\text {contact }[i]} \triangleq p_{\text {contact }[i]}-p_{\text {wheel }[i]}$ is the displacement from the wheel center to the contact point. The rolling constraint implies that the velocity of the contact point in the direction of the wheel is zero, i.e. $u_{\text {rolling }[i]}^{T} v_{\operatorname{contact}[i]}=$ 0 , where $u_{\text {rolling }[i]} \in \mathbb{R}^{3}$ is the direction of the wheel axis. We rewrite this condition as $J_{\text {rolling }[i]} \dot{q}=0$, where

$$
J_{\text {rolling }[i]} \triangleq u_{\text {rolling }[i]}^{T}\left(J_{\omega, \text { wheel }[i]}-\delta_{\text {contact }[i]} \times J_{\omega, \text { wheel }[i]}\right)
$$

and $J_{\text {wheel }[i]} \triangleq\left(\begin{array}{ll}J_{\mathrm{v} \text {, wheel }[i]}^{T} & \left.J_{\omega, \text { wheel }[i]}^{T}\right)^{T} \in \mathbb{R}^{6 \times n_{\text {dofs }}} \text { are }\end{array}\right.$ the linear and rotational part of the wheel's basic Jacobian.

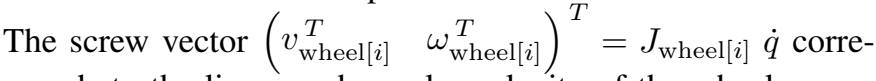
sponds to the linear and angular velocity of the wheel.

In addition, there is a contact constraint with respect to the wheel's normal direction to the ground: $J_{\text {normal }[i]} \dot{q}=0$, with $J_{\text {normal }[i]} \triangleq u_{\text {normal }[i]}^{T} J_{\text {wheel }}$. Therefore the combined rolling constraints of the three wheels correspond to the aggregation of the slip and the normal constraints for each wheel, i.e.

$$
J_{\text {contact }} \triangleq\left(\begin{array}{c}
J_{\text {rolling[1] }} \\
J_{\text {rolling[2] }} \\
J_{\text {rolling[3] }} \\
J_{\text {normal[1] }} \\
J_{\text {normal[2] }} \\
J_{\text {normal[3] }}
\end{array}\right) \in \mathbb{R}^{6 \times n_{\text {dofs }}}
$$

\section{Compliant Whole-Body Control}

The whole-body control framework expresses controllers in a representation that is the most natural for a given task, with a

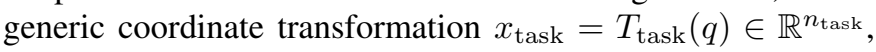
e.g. a homogeneous transformation for a Cartesian point, or a rotation and translation in the group $S E(3)$ for a spatial transformation of a frame. It follows that instantaneous task kinematics can be expressed as $\dot{x}_{\text {task }}=J_{\text {task }} \dot{q}$, where $J_{\text {task }}=$ $\partial x_{\text {task }} / \partial q \in \mathbb{R}^{n_{\text {task }} \times n_{\text {dofs }}}$ is the geometric Jacobian matrix with respect to the generalized coordinates.

In order to express the task with respect to the actuated joints, section III-A summarizes earlier results on a dynamically consistent generalized inverse of $U N_{\text {constr. Then, }}$. section III-B proceeds to develop a prioritized operational space task/posture controller for Dreamer, and section III-C contributes a new proof of stability for our posture control approach.

The sum of all tasks may not fully determine the motor torques for all actuated joints. In fact, this is a very common circumstance with redundant manipulators such as Dreamer. In order to stabilize the motions that remain in the null space of all tasks, we define posture as a special kind of task which optimize a performance criterion, as opposed to the usual position or force tracking. We usually place at least one posture at the end of any task hierarchy.

\section{A. Actuated-Joint Kinematics}

The number of constraints (one for the biarticulate joint plus two for each of the three wheels in contact) is equal to the 
number of unactuated DOF (one for the biarticulate joint and six for the free-floating formulation). Thus, we can solve the robot's kinematics from the actuated joints alone. In particular, we consider decomposing the generalized coordinates into actuated and unactuated parts, i.e.

$$
q=D_{\text {kin }}\left(\begin{array}{c}
q_{\text {act }} \\
q_{\text {unact }}
\end{array}\right)
$$

where $D_{\text {kin }}$ is a matrix that distributes generalized coordinates to their respective positions in the robot's kinematic chain.

Because of the already encountered condition $J_{\text {constr }} \dot{q}=0$, we can state that $\dot{q}^{*} \in \operatorname{Null}\left(J_{\text {constr }}\right)$ where $\dot{q}^{*}$ expresses the set of generalized velocities that fulfills all of the constraints. In other words, the constrained velocities can be expressed as the self motion manifold $\dot{q}^{*}=\left(I-J_{\text {constr }}^{\#} J_{\text {constr }}\right) \dot{q}$, where $J_{\text {constr }}^{\#}$ is any right inverse of the constraint Jacobian (i.e. $J_{\text {constr }} J_{\text {constr }}^{\#}=I$ ) and $\dot{q}$ represents unconstrained velocities.

The constrained generalized velocities can be reconstructed from the velocities of the actuated DOF alone using the following formula

$$
\dot{q}^{*}=\overline{U N}_{\text {constr }} \dot{q}_{\text {act }},
$$

where

$$
\overline{U N}_{\text {constr }} \triangleq A^{-1}\left(U N_{\text {constr }}\right)^{T}\left(\phi^{*}\right)^{+},
$$

is the dynamically consistent generalized inverse of $U N_{\text {constr }}$, $(.)^{+}$is the Moore-Penrose pseudoinverse operator and

$$
\phi^{*} \triangleq U N_{\text {constr }} A^{-1}\left(U N_{\text {constr }}\right)^{T},
$$

is a projection of the inertia matrix in the reduced constrained manifold. See [20] for a proof of (13).

\section{B. Prioritized Control Structure}

Based on (14), the differential kinematics can now be expressed with respect to actuated joints alone:

$$
\dot{x}_{\text {task }}=J_{\text {task }}^{*} \dot{q}_{\text {act }},
$$

where $J_{\text {task }}^{*}$ is the reduced constraint consistent Jacobian

$$
J_{\text {task }}^{*} \triangleq J_{\text {task }} \overline{U N}_{\text {constr }}
$$

with $J_{\text {task }}^{*} \in \mathbb{R}^{n_{\text {task }} \times n_{\text {act }}}$ a reduced form of the task Jacobian consistent with the general constraint conditions. To prove (16), consider the constrained generalized velocities $\dot{q}^{*}$, and apply (13) to the instantaneous task kinematics, thus getting $\dot{x}_{\text {task }}=J_{\text {task }} \dot{q}^{*}=J_{\text {task }} \overline{U N}_{\text {constr }} \dot{q}_{\text {act }}$.

Now, consider the skill of touching objects using our robot, encoded as a control hierarchy with one task and one posture. This skill uses two coordinate systems, $x_{\text {skill }} \triangleq\left\{x_{\text {hand }} \in\right.$ $\left.T(3), x_{\text {posture }}=q_{\text {act }} \in \mathbb{R}^{n_{\text {act }}}\right\}$, where $T(3)$ is the group of translations. The experiments in section IV employ a controller which follows the same principle, but it adds a balancing task to the top of the control hierarchy.

In general, an arbitrary task $(k)$ can be kinematically characterized by its mapping with respect to the generalized coordinates and by its generalized constrained Jacobian, i.e.

$$
\begin{aligned}
& x_{\operatorname{task}(k)}=T_{\operatorname{task}(k)}(q), \\
& \dot{x}_{\operatorname{task}(k)}=J_{\operatorname{task}(k)}^{*} \dot{q}_{\text {act }},
\end{aligned}
$$

$$
\text { with } \quad J_{\operatorname{task}(k)}^{*} \triangleq J_{\operatorname{task}(k)} \overline{U N}_{\text {constr }} \text {. }
$$

It can be demonstrated [20], that the dynamics of the actuated joints can be expressed as

$$
\ddot{q}_{\text {act }}+\phi^{*}\left(b^{*}+g^{*}\right)=\phi^{*} \tau_{\text {control }} .
$$

where

$\phi^{*}\left(b^{*}+g^{*}\right) \triangleq U A^{-1}\left(N_{\text {constr }}^{T}(b+g)+J_{\text {constr }}^{T} \Lambda_{\text {constr }} \dot{J}_{\text {constr }} \dot{q}\right)$.

By left multiplying the constrained actuated dynamics by $\bar{J}_{\operatorname{task}(k)}^{*} \triangleq \phi^{*} J_{\operatorname{task}(k)}^{T}\left(J_{\operatorname{task}(k)} \phi^{*} J_{\operatorname{task}(k)}^{T}\right)^{-1}$, and using the equality, $\ddot{x}_{\operatorname{task}(k)}=J_{\operatorname{task}(k)}^{*} \ddot{q}_{\text {act }}+\dot{J}_{\operatorname{task}(k)}^{*} \dot{q}_{\text {act }}$, the constrained task dynamics can be expressed as

$$
\Lambda_{\operatorname{task}(k)}^{*} \ddot{x}_{\operatorname{task}(k)}+\mu_{\operatorname{task}(k)}^{*}+p_{\operatorname{task}(k)}^{*}=\bar{J}_{\operatorname{task}(k)}^{* T} \tau_{\text {control }},
$$

with $\left\{\Lambda_{\operatorname{task}(k)}^{*}, \mu_{\operatorname{task}(k)}^{*}, p_{\operatorname{task}(k)}^{*}\right\}$ being inertial, Corioliscentrifugal, and gravitational terms (not derived here).

If $J_{\operatorname{task}(k)}^{*}$ is full rank, the following control structure yields full control of the task dynamics,

$$
\tau_{\text {control }}=J_{\operatorname{task}(k)}^{* T} F_{\operatorname{task}(k)} .
$$

This statement can be proven by applying the above torques into (23) and using the property of the generalized inverse $J_{\operatorname{task}(k)}^{*} \bar{J}_{\operatorname{task}(k)}^{*}=I$, thus getting

$$
\Lambda_{\operatorname{task}(k)}^{*} \ddot{x}_{\operatorname{task}(k)}+\mu_{\operatorname{task}(k)}^{*}+p_{\operatorname{task}(k)}^{*}=F_{\operatorname{task}(k)},
$$

which delivers full control of the task dynamics $\ddot{x}_{\operatorname{task}(k)}$ by using feedforward control and feedback linearization by means of the control input $F_{\operatorname{task}(k)}$, i.e.

$$
F_{\operatorname{task}(k)}=\Lambda_{\operatorname{task}(k)}^{*} a_{\operatorname{task}(k)}^{\mathrm{ref}}+\mu_{\operatorname{task}(k)}^{*}+p_{\operatorname{task}(k)}^{*},
$$

where $a_{\operatorname{task}(k)}^{\mathrm{ref}}$ is an acceleration control input. The above controller yields $\ddot{x}_{\operatorname{task}(k)}=a_{\operatorname{task}(k)}^{\mathrm{ref}}$.

For the case of one task and one posture, we define the following prioritized control structure that provides optimal projection of the posture gradient,

$$
\tau_{\text {control }}=J_{\text {task }}^{* T} F_{\text {task }}+N_{\text {task }}^{* T} \tau_{\text {posture }},
$$

where $N_{\text {task }}^{*} \triangleq I-\bar{J}_{\text {task }}^{*} J_{\text {task }}^{*}$, is the dynamically consistent null-space matrix of the reduced task Jacobian.

\section{Posture Control Stability}

Uncontrolled null-space tasks are known to produce unstable behaviors [5]. Therefore, we investigate the stabilizing properties of feedback controllers for the posture. We consider the following energy function to be minimized in posture space

$$
V_{\text {posture }}=\frac{1}{2} e_{q_{\text {act }}}^{T} K e_{q_{\text {act }}},
$$

where $e_{q_{\text {act }}} \triangleq q_{\text {act }}-q_{\text {act }}^{\text {goal }}$ is a feedback error function, $q_{\text {act }}^{\text {goal }}$ is a postural attractor goal and $K$ is a gain matrix. A naïve approach is to project the gradient of (28) directly into the control structure (27), i.e.

$$
\tau_{\text {control }}=J_{\text {task }}^{* T} F_{\text {task }}+N_{\text {task }}^{* T} K e_{q_{\text {act }}} .
$$




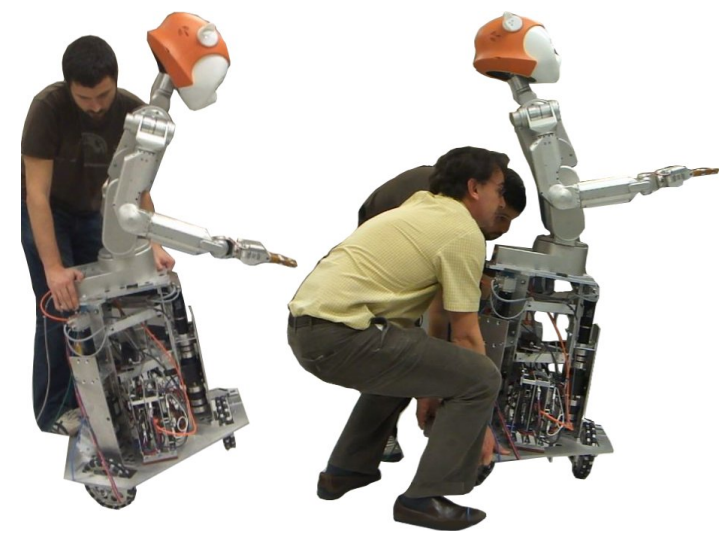

Fig. 3. Balance: The upper body is controlled such that, when projected on to a horizontal plane, its center of mass (COM) remains as close as possible to the projected center of the support triangle formed by the three wheels. This is demonstrated by manually tilting the base. Notice that the COM task controls two DOF, and the posture control takes care of stabilizing the joints at a position that minimizes the posture error while not interfering with the projected COM position.

If we apply the above torque to the posture dynamics (which are equal to the actuated dynamics) of Equation (21), and neglect the task torques, i.e. $F_{\text {task }}=0$, we get $\ddot{q}_{\text {act }}+$ $\phi^{*}\left(b^{*}+g^{*}\right)=\phi^{*} N_{\text {task }}^{* T} K e_{q_{\text {act }}}$.

The problem with this policy is that if the gradient of the energy fulfills $K e_{q_{\text {act }}} \in \operatorname{Null}\left(N_{\text {task }}^{* T}\right)$, then convergence to the minimum energy is not possible. It is said that the joint error $e_{q_{\text {act }}}$ gets "stuck" in the null space of $N_{\text {task }}^{* T}$. This type of deficiency has been studied before, for instance in [18] by proposing a time varying feedback stabilization scheme which augments the gain matrix $K$. However, such solution does not take into account the null space characteristics, and therefore cannot optimize the gradient descent. In [15], [21], we extensively investigated this problem in the context of dynamic behavior of posture control, and proposed a control strategy equivalent to choosing a gain matrix that rotates the gradient to the best possible direction away from $\operatorname{Null}\left(N_{\text {task }}^{* T}\right)$. It was found that the following postural control structure yields favorable gradient descent of postural energies

$$
\tau_{p \mid t}=J_{p \mid t}^{* T} F_{\text {posture }}
$$

where $\tau_{p \mid t} \triangleq N_{\text {task }}^{* T} \tau_{\text {posture }}$ is the right-most term of (27),

$$
J_{p \mid t}^{*} \triangleq J_{\text {posture }}^{*} N_{\text {task }}^{*}
$$

is the task consistent posture Jacobian matrix and $J_{\text {posture }}^{*} \triangleq$ $U \overline{U N}_{\text {constr }}$, is the reduced constraint consistent Jacobian of the posture. To prove (30) let us consider the following steps: 1. If we plug (30) into (21) we get

$$
\ddot{q}_{\text {act }}+\phi^{*}(b+g)=\phi^{*} J_{p \mid t}^{* T} F_{\text {posture }} .
$$

However, in [20] it was demonstrated that the right hand terms $\phi^{*} J_{p \mid t}^{* T}=\phi_{p \mid t}^{*}$, where

$$
\phi_{p \mid t}^{*} \triangleq J_{p \mid t}^{*} \phi^{*} J_{p \mid t}^{* T},
$$

is an inverse inertial matrix in a space that is simultaneously consistent with the robot constraints and with the task priority.
2. As such (32) becomes

$$
\ddot{q}_{\text {act }}+\phi^{*}\left(b^{*}+g^{*}\right)=\phi_{p \mid t}^{*} F_{\text {posture }}
$$

By choosing the feedback control policy

$$
F_{\text {posture }}=\left(\phi_{p \mid t}^{*}\right)^{+} K e_{q_{\text {act }}}+\left(\phi_{p \mid t}^{*}\right)^{+} \phi^{*}\left(b^{*}+g^{*}\right),
$$

leads to [20]

$$
U_{\text {rank }, p \mid t}^{T}\left(\ddot{q}_{\text {act }}=K e_{q_{\text {act }}}\right)
$$

where

$$
\phi_{p \mid t}^{*}=U_{p \mid t}^{\mathrm{rank}} \Sigma_{p \mid t}^{\mathrm{rank}}\left(U_{p \mid t}^{\mathrm{rank}}\right)^{T}
$$

is the minimal (rank) svd decomposition of $\phi_{p \mid t}^{*}$, and

$$
U_{p \mid t}^{\mathrm{rank}} \in \mathbb{R}^{n_{\text {act }} \times\left(n_{\text {act }}-n_{\text {task }}\right)},
$$

is the set of controllable eigenvectors of $\phi_{p \mid t}^{*}$.

The feedback behavior of (36) implies that we are descending the gradient in the controllable directions left out by $N_{\text {task }}^{* T}$. The resulting performance improvement is substantial [15].

As a new contribution, we study in more detail the stability of self-motions of our proposed controller. The general controller $F_{\text {posture }}=\left(\phi_{p \mid t}^{*}\right)^{+} v+\left(\phi_{p \mid t}^{*}\right)^{+} \phi^{*}\left(b^{*}+g^{*}\right)$ yields the closed loop behavior [20]

$$
\ddot{q}_{\text {act }}=\Phi_{p \mid t}^{*} v
$$

where $\Phi_{p \mid t}^{*} \triangleq \phi_{p \mid t}^{*} \phi_{p \mid t}^{*+}=U_{p \mid t}^{\mathrm{rank}}\left(U_{p \mid t}^{\mathrm{rank}}\right)^{T}$ is a singular and symmetric positive semidefinite matrix that represents the self motion manifold of the task coordinates, and $v$ a closed loop controller. Let us define a projected state error

$$
E \triangleq \Phi_{p \mid t}^{*}\left(-k_{p} e_{\text {act }}-k_{v} \dot{q}_{\text {act }}\right)
$$

with $k_{p}>0, k_{v}>0$ and $e_{\text {act }} \triangleq q_{\text {act }}-q_{\text {act }}^{\text {goal }}$. The controller choice

$$
v=\frac{1}{k_{v}} K_{E} E-\frac{k_{p}}{k_{v}} \dot{q}_{\text {act }}-\frac{1}{k_{v}} \dot{\Phi}_{p \mid t}^{*}\left(-k_{p} e_{\text {act }}-k_{v} \dot{q}\right),
$$

where $K_{E}$ is a gain matrix, will render stable behavior of the self-motions. To demonstrate the stability, let us consider the Lyapunov candidate function

$$
V=\frac{1}{2} E^{T} E \geq 0 .
$$

In particular it can be made strictly positive definite by choosing error coordinates $e_{\text {act }}$ away from the null eigenvectors. In fact, it would be almost impossible to choose an error vector that falls in the null eigenvectors since desired postures are uncorrelated with the task. The time derivative yields

$$
\begin{aligned}
\dot{V}= & E^{T} \dot{E}=-E^{T}\left(k_{p} \dot{q}_{\text {act }}+k_{v} v\right) \\
& -E^{T} \dot{\Phi}_{p \mid t}^{*}\left(k_{p} e_{\text {act }}+k_{v} \dot{q}_{\text {act }}\right)=-E^{T} K_{E} E \leq 0
\end{aligned}
$$

where we have used the equality

$$
\begin{aligned}
& \dot{E}=\Phi_{p \mid t}^{*}\left(-k_{p} \dot{q}_{\mathrm{act}}-k_{v} \ddot{q}_{\mathrm{act}}\right)+\dot{\Phi}_{p \mid t}^{*}\left(-k_{p} e_{\mathrm{act}}-k_{v} \dot{q}_{\mathrm{act}}\right) \\
& =\Phi_{p \mid t}^{*}\left(-k_{p} \dot{q}_{\mathrm{act}}-k_{v} \Phi_{p \mid t}^{*} v\right)+\dot{\Phi}_{p \mid t}^{*}\left(-k_{p} e_{\mathrm{act}}-k_{v} \dot{q}_{\mathrm{act}}\right)
\end{aligned}
$$




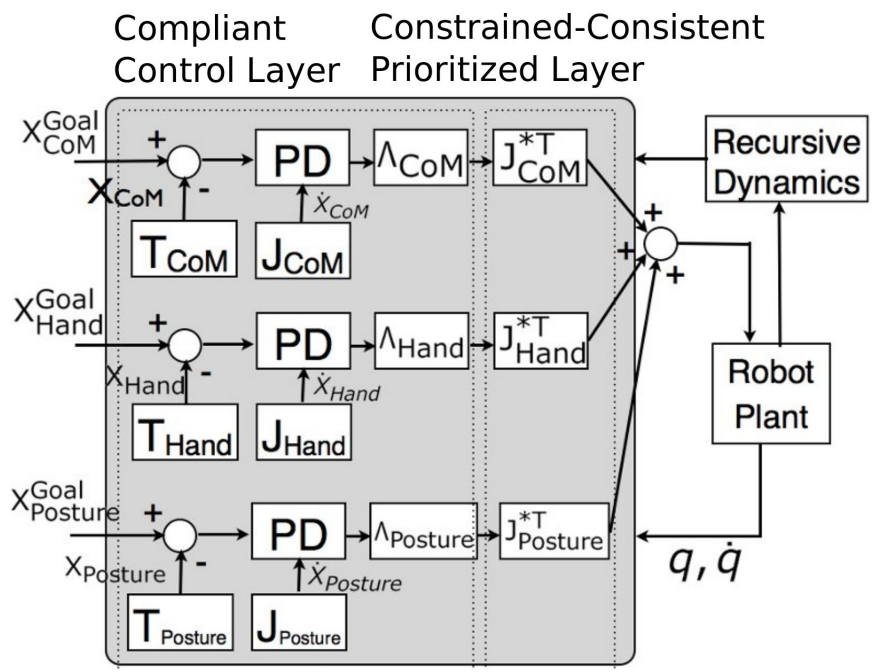

Fig. 4. Prioritized whole-body compliant controller.

the property of idempotence $\Phi_{p \mid t}^{*} \Phi_{p \mid t}^{*}=\Phi_{p \mid t}^{*}$ (which follows from the property of generalized inverse $\left.\phi_{p \mid t}^{*} \phi_{p \mid t}^{*+} \phi_{p \mid t}^{*}=\phi_{p \mid t}^{*}\right)$ and the closed loop input of (41). Because Equation (43) is essentially the negative equivalent of Equation (42), the proposed self-motion controller renders stable behavior of the posture if (42) is chosen to be striclty positive.

Tracking under model uncertainties remains an open question for our posture controller. We are planning to conduct such an analysis inspired by [4], [23], [10].

\section{EXPERIMENTS}

We performed an experiment involving maneuvering in an irregular terrain while physically interacting with a human user. It serves as empirical validation for the approach, by focusing on one essential irregular terrain feature, namely a change in slope between two planar surfaces.

This relatively simple setup, shown in Figure 1 already contains many of the challenges that Dreamer will have to face on more irregular terrain: excessive tilting or upper body accelerations may topple the robot; feed-forward gravity compensation, which is crucial for accurate yet compliant task control, depends on orientation; and forces due to interaction with the environment can lift wheels off the ground or lead to unwanted slippage of the base.

The objective of the experiment is to demonstrate that: (1) the robot can transition safely between the flat and the sloping portions at various speeds; (2) the IMU unit and the models of the robot produce a good estimation of the constrained freebody dynamics; (3) the base follows the body as a result of the compliant interactions and because of the contact models; (4) the robot can control balance to prevent falling; (5) accurate manipulation and physical interaction can take place in the irregular terrain; (6) posture energies are minimized given the control objectives; and (7) all of this is accomplished while complying with the biarticular and rolling/contact constraints.

To show the desired capabilities, we create a skill for interacting with a human user in the irregular terrain. It is very similar to the one discussed in section III-B, with two modifications.
First of all, a balance task prevents falling when transitioning from flat to sloping terrain. This task controls the position of the center of mass (COM) projected onto a horizontal plane. Second, the posture is modified to act only in the upper body DOF, which naturally implements kinesthetic control of the base: a human user simply needs to gently push or pull the hand in the direction where the robot should go, and without further modifications to the system model or the control formulation, the three base DOF will provide the required motion. This works because the whole-body formulation includes all DOF in all tasks, and the hand goal is defined relative to the body, thus moving the base closer to the hand.

The coordinates of this Phyisical Human-Robot Interaction (pHRI) skill are thus $x_{\text {skill }}(q) \triangleq\left\{x_{\text {com }}, x_{\text {hand }}, x_{\text {posture }}\right\} \in$

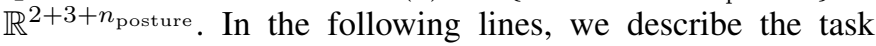
kinematics and then formulate the prioritized whole-body control structure. All the controllers and related software were implemented using the open source realtime software infrastructure described in [19].

The balance task coordinates are

$$
x_{\mathrm{com}} \triangleq \frac{1}{M} \sum_{i=i_{\mathrm{base}}}^{n_{\mathrm{dofs}}} m_{i} x_{\mathrm{com}[i]}
$$

where $M$ is the total mass of the robot, $i_{\text {base }}=n_{\text {freebody }}+$ $n_{\text {wheels }}$ is the first joint attached to the upper body base in the kinematic chain depicted in Figure 2. The COM Jacobian can thus be given as

$$
J_{\text {com }}(q)=\frac{1}{M} \sum_{i=i_{\text {base }}}^{n_{\text {dofs }}} m_{i} J_{\text {com }[i]}(q)
$$

where $J_{\operatorname{com}[i]}$ is the Jacobian of the $i$-th linkage in the kinematic chain.

The hand coordinate system can be derived from the homogeneous transformation from the Inertial Frame $\mathcal{I}$ to the Hand Frame $\mathcal{H}$, both depicted in Figure 2. Thus, the hand frame is given by

$$
\left(\begin{array}{c}
x_{\text {hand }} \\
1
\end{array}\right)=\prod_{i=0}^{n_{\text {hand }}}{ }^{i+1} T_{i}(q)\left(\begin{array}{c}
\mathcal{H} \\
x_{\text {hand }} \\
1
\end{array}\right),
$$

where $x_{\text {hand }}$ is the vector of hand translations with respect to the inertial frame $\mathcal{I},{ }^{i+1} T_{i}(q)$ is the matrix describing the Homogeneous Transformation from frame $i+1$ to frame $i$, and ${ }^{\mathcal{H}} x_{\text {hand }}$ is the hand translation expressed in its local frame $\mathcal{H}$. The Jacobian of the hand is thus the geometric operator $J_{\text {hand }}(q) \triangleq \partial x_{\text {hand }} / \partial q$.

The posture coordinates correspond to the actuated joints of the upper body. Notably, for reasons described above, the wheels are not included. Therefore $x_{\text {posture }}=S_{\text {act }}^{\text {upper }} q$, where $S_{\text {act }}^{\text {upper }}$ is a selection matrix corresponding to the actuated joints of the upper body only. It follows that the posture Jacobian is $J_{\text {posture }}=S_{\text {act }}^{\text {upper }}$.

The prioritized whole-body compliant controller described earlier endows Dreamer with the pHRI skill for this experiment. The control structure can be expressed as

$$
\tau_{\text {control }}=J_{\text {com }}^{* T} F_{\text {com }}+J_{\text {hand }}^{* T} F_{\text {hand }}+J_{\text {posture }}^{* T} F_{\text {posture }},
$$



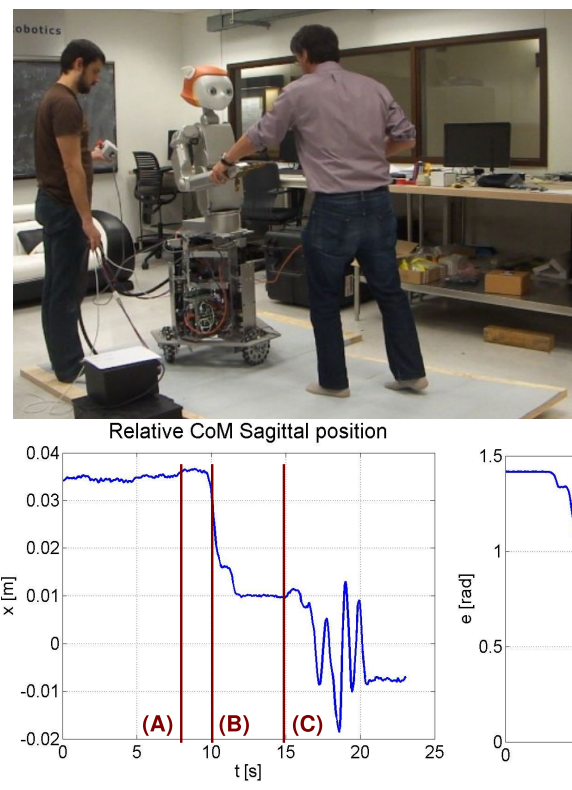

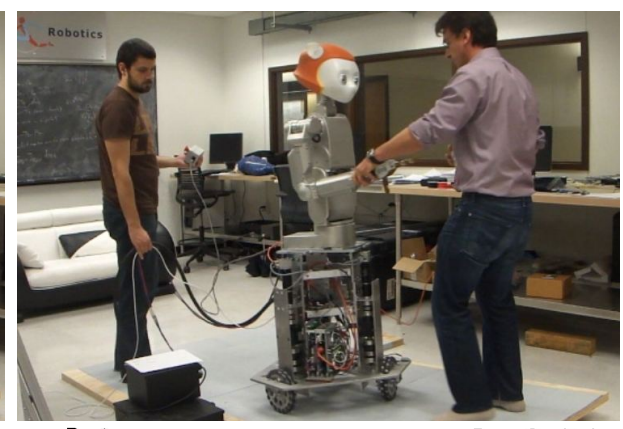

Posture error

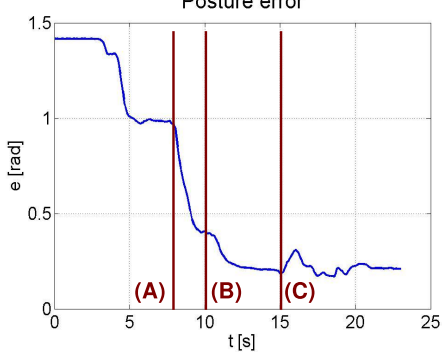

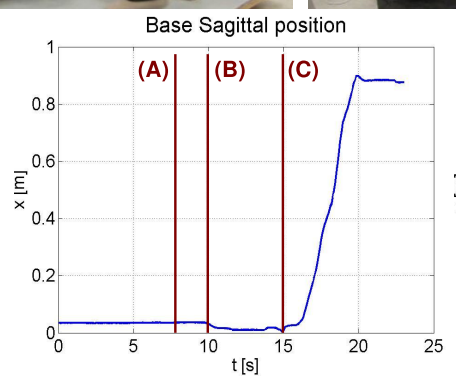

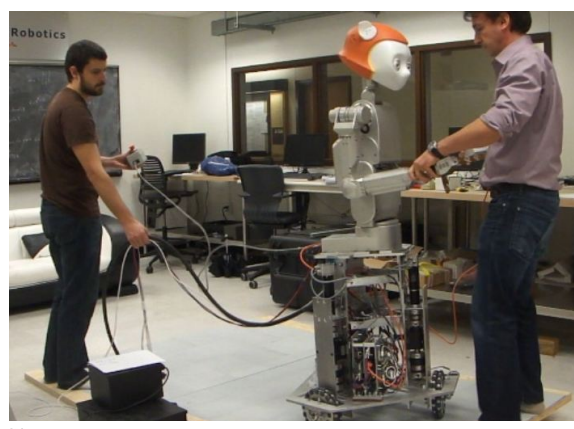

Relative hand Sagittal position

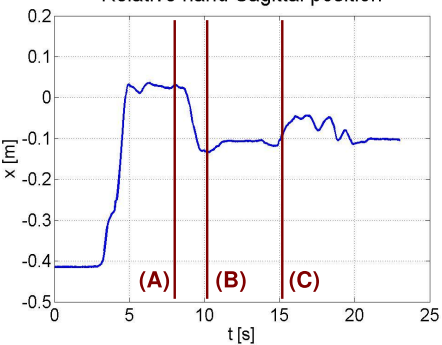

Fig. 5. Experimental Results. Sequence of video snapshots, and data taken during the experiment on the irregular terrain. At instant (A) the upper body motors are enabled. At (B) the base motors are enabled. At (C) the robot starts ascending the slope.

where the center of mass operates with higher priority than the hand task and the hand task operates with higher priority than the posture task. Priorities are enforced by recursively defining the differential manifold of the self-motion of higher priority tasks, i.e.

$$
\begin{aligned}
& J_{\text {com }}^{*}=J_{\text {com }} \overline{U N}_{\text {constr }}, \\
& J_{\text {hand }}^{*}=J_{\text {hand }} \overline{U N}_{\text {constr }} N_{\text {com }}^{*}, \\
& J_{\text {posture }}^{*}=J_{\text {posture }} \overline{U N}_{\text {constr }} N_{\text {hand,com }}^{*},
\end{aligned}
$$

with null spaces of self-motion expressed as [20]

$$
\begin{aligned}
& N_{\text {com }}^{*}=I-\bar{J}_{\text {com }}^{*} J_{\text {com }}^{*}, \\
& N_{\text {hand,com }}^{*}=I-\bar{J}_{\text {hand }}^{*} J_{\text {hand }}^{*}-\bar{J}_{\text {com }}^{*} J_{\text {com }}^{*} .
\end{aligned}
$$

The above control structures leverage the developments in sections II and III to achieve dynamic consistency. $F_{\text {com }}$, $F_{\text {hand }}$, and $F_{\text {posture }}$ constitute the input commands.

We now focus on the feedback control policies for the overall controller. Figure 4 shows the flow diagram. The goal of the COM task is to move the robot's center of mass to a goal position above the center of the triangle defined by the wheels (see Figure 3 ). The goal of the hand task is to reach a position in front of the robot's body, compliance with respect to the human is achieved by reducing the control gains. The posture goal is to move the upper body actuated joints to the zero position, except for the elbow joint which should bend $90^{\circ}$. Summarizing,

$$
\begin{aligned}
& x_{\mathrm{com}}^{\text {goal }}=\text { projected center of the base } \in \mathbb{R}^{2} \\
& x_{\text {hand }}^{\text {goal }}=\text { position relative to body } \in \mathbb{R}^{3} \\
& x_{\text {posture }}^{\text {gool }}=\text { posture with bent elbow } \in \mathbb{R}^{n_{\text {upperbody }}-1} .
\end{aligned}
$$

We assume that the center of mass and hand tasks are nonsingular in their priority spaces. Therefore, the objectives of these tasks are to converge asymptotically to the goal. In contrast, the posture is required to be locally stable only. The proposed feedback/feedforward control laws are

$$
\begin{aligned}
& F_{\text {com }}=\Lambda_{\text {com }}^{*}\left(-k_{p, \text { com }} e_{\text {com }}^{\text {goal }}-k_{v, \text { com }} \dot{x}_{\text {com }}\right)+p_{\text {com }} \\
& F_{\text {hand }}=\Lambda_{\text {hand }}^{*}\left(-K_{p, \text { hand }} e_{\text {hand }}^{\text {goal }}-K_{v, \text { hand }} \dot{x}_{\text {hand }}\right)+p_{\text {hand }}
\end{aligned}
$$

$F_{\text {posture }}=$

$\Lambda_{\text {posture }}^{*}\left(-k_{p, \text { posture }} e_{\text {posture }}^{\text {goal }}-k_{v, \text { posture }} \dot{x}_{\text {posture }}\right)+p_{\text {posture }}$.

Given the above linearizing control laws, the resulting closedloop dynamics of the task coordinates become

$$
\begin{aligned}
& \ddot{x}_{\mathrm{com}}+k_{p, \mathrm{com}} e_{\mathrm{com}}^{\text {goal }}+k_{v, \mathrm{com}} \dot{x}_{\mathrm{com}}=0, \\
& \ddot{x}_{\text {hand }}+K_{p, \text { hand }} e_{\text {hand }}^{\text {goal }}+K_{v \text {, hand }} \dot{x}_{\mathrm{com}}=0,
\end{aligned}
$$

which are globally asymptotically stable while the closed-loop dynamics of the posture become

$$
U_{\mathrm{r}, \text { posture }}^{T}\left[\ddot{x}_{\text {posture }}+k_{p, \text { posture }} e_{\text {com }}^{\text {goal }}+k_{v, \text { posture }} \dot{x}_{\text {com }}\right]=0 \text {, }
$$

which is locally stable.

Note that there are alternative approaches for creating such a skill. For example, inverse kinematic [22], Resolved Momentum [12], whole-body impedance controller [7]. To the best of our knowledge, however, none of these is adept at handling irregular terrains.

Figure 5 shows a sequence of snapshots and the accompanying data of the experiment. The robot starts on flat terrain. Then, the human operator pulls gently backward on the hand, and the robot transitions to the slope. After approximately 1 meter, the robot is pushed back to the horizontal area. Looking at the data plots, various phases can be discerned.

During the first 8 seconds, the emergency stop button was engaged while the operator moved from the command inter- 
face to the robot. Fluctuations of hand position and postural error are due to the operator grasping Dreamer's upper arm.

After 8 seconds, the emergency button of the upper body is released, the hand position stabilizes and the postural error gets minimized.

The independent power of the base is turned on after $10 \mathrm{sec}-$ onds. Consequently, the COM relative error gets minimized.

After 15 seconds, the operator starts to guide the robot up the slope. It can be seen that the base moves for approximately $0.9 \mathrm{~m}$ at a speed of about $0.35 \mathrm{~m} / \mathrm{s}$. During this time, the balance task maintains the COM within $1 \mathrm{~cm}$ of its goal. The relative hand position also remains stable and close to its goal. Note that the operator keeps pulling on the hand during all of this, and notice that the postural error is minimized in the task's null space throughout the interaction.

\section{CONCLUSION}

Controlling mobile humanoid robots in irregular terrains entails modeling the free-body dynamics constrained by wheel contacts and, in our case, a biarticular transmission in the torso. Based on these foundations, we have presented a prioritized compliant controller which precisely tracks a hierarchy of tasks and optimizes a posture in the remaining degrees of redundancy. The general applicability of our approach has been demonstrated with a detailed study of closed loop stability and an experiment that reflects some of the key challenges while remaining sufficiently simple to serve as an illustrative example.

A remarkable result is that base movement can emerge quasi spontaneously, adapting to the terrain without an explicit representation of the feasible directions. This is enabled by the properly modeled contact constraints and using an IMU to estimate spatial orientation. Another important consequence is that we do not require an explicit model of the Omni wheels with respect to the desired direction of motion.

The whole-body skill used in the experiment is simple yet powerful, which demonstrates the synergistic capabilities of the framework and the robot. Firstly, the posture behavior coupled with the compliant hand task ensures that the robot kinesthetically follows the human around the terrain. Secondly, the IMU sensor feedback and the balance task ensure that the center of mass always stays very close to the optimal location, even while transitioning between horizontal and sloped sections. Lastly, tracking and optimizing the various goals takes advantage of all degrees of freedom and constraints.

Future directions of this work include, detecting wheel contacts due to disturbances with terrain debris, dealing with wheel traction and slip, and improving the hardware to reduce actuator friction, transmission alignment, backlash and shock absortion.

\section{REFERENCES}

[1] O. Brock, O. Khatib, and S. Viji. Task-consistent obstacle avoidance and motion behavior for mobile manipulation. In Proceedings of the IEEE International Conference on Robotics and Automation, pages 388-393, Washingtion, USA, 2002.

[2] H. Bruyninckx. Open robot control software: the orocos project. In Robotics and Automation, 2001. Proceedings 2001 ICRA. IEEE International Conference on, volume 3, pages 2523 - 2528 vol.3, 2001.
[3] K.S. Chang, R. Holmberg, and O. Khatib. The augmented object model: Cooperative manipulation and parallel mechanism dynamics. In Robotics and Automation, 2000. Proceedings. ICRA'O0. IEEE International Conference on, volume 1, pages 470-475. IEEE, 2000.

[4] CC Cheah, C. Liu, and JJE Slotine. Adaptive tracking control for robots with unknown kinematic and dynamic properties. The International Journal of Robotics Research, 25(3):283-296, 2006.

[5] A. DeLuca. Zero dynamics in robotics systems. Progress in Systems and Control Theory, Proceedings of the IIASA Workshop, 9:70-87, 1991.

[6] A. Dietrich, T. Wimbock, and A. Albu-Schaffer. Dynamic wholebody mobile manipulation with a torque controlled humanoid robot via impedance control laws. In Intelligent Robots and Systems (IROS), 2011 IEEE/RSJ International Conference on, pages 3199 -3206, sept. 2011.

[7] A. Dietrich, T. Wimbock, and A. Albu-Schaffer. Dynamic wholebody mobile manipulation with a torque controlled humanoid robot via impedance control laws. In Intelligent Robots and Systems (IROS), 2011 IEEE/RSJ International Conference on, pages 3199-3206. IEEE, 2011.

[8] Gustavo Freitas, Fernando Lizarralde, Liu Hsu, and Ney R. Salvi dos Reis. Kinematic reconfigurability of mobile robots on irregular terrains. In Robotics and Automation, 2009. ICRA '09. IEEE International Conference on, pages 1340 -1345, may 2009.

[9] M. Fuchs, C. Borst, P.R. Giordano, A. Baumann, E. Kraemer, J. Langwald, R. Gruber, N. Seitz, G. Plank, K. Kunze, R. Burger, F. Schmidt, T. Wimboeck, and G. Hirzinger. Rollin' justin - design considerations and realization of a mobile platform for a humanoid upper body. In Robotics and Automation, 2009. ICRA '09. IEEE International Conference on, pages $4131-4137$, may 2009.

[10] M. Galicki. An adaptive regulator of robotic manipulators in the task space. Automatic Control, IEEE Transactions on, 53(4):1058-1062, 2008.

[11] Robert Holmberg and Oussama Khatib. Development and control of a holonomic mobile robot for mobile manipulation tasks. The International Journal of Robotics Research, 19(11):1066-1074, 2000.

[12] S. Kajita, F. Kanehiro, K. Kaneko, K. Fujiwara, K. Harada, K. Yokoi, and H. Hirukawa. Resolved momentum control: Humanoid motion planning based on the linear and angular momentum. In Proceedings of the IEEE/RSJ International Conference on Intelligent Robots and Systems, pages 1644-1650, Las Vegas, USA, October 2003.

[13] Fumio Kanehiro, Hirohisa Hirukawa, and Shuuji Kajita. Openhrp: Open architecture humanoid robotics platform. The International Journal of Robotics Research, 23(2):155-165, 2004.

[14] O. Khatib. A unified approach for motion and force control of robot manipulators: The operational space formulation. International Journal of Robotics Research, 3(1):43-53, 1987.

[15] O. Khatib, L. Sentis, J.H. Park, and J. Warren. Whole body dynamic behavior and control of human-like robots. International Journal of Humanoid Robotics, 1(1):29-43, March 2004.

[16] O. Matsumoto, S. Kajita, K. Tani, and M. Oooto. A four-wheeled robot to pass over steps by changing running control modes. In Robotics and Automation, 1995. Proceedings., 1995 IEEE International Conference on, volume 2, pages 1700-1706. IEEE, 1995.

[17] K. Nagasaka, Y. Kawanami, S. Shimizu, T. Kito, T. Tsuboi, A. Miyamoto, T. Fukushima, and H. Shimomura. Whole-body cooperative force control for a two-armed and two-wheeled mobile robot using generalized inverse dynamics and idealized joint units. In Robotics and Automation (ICRA), 2010 IEEE International Conference on, pages $3377-3383$, may 2010.

[18] G. Oriolo. Stabilization of self-motions in redundant robots. In Robotics and Automation, 1994. Proceedings., 1994 IEEE International Conference on, pages 704-709. IEEE, 1994.

[19] R. Philippsen, L. Sentis, and O. Khatib. An open source extensible software package to create whole-body compliant skills in personal mobile manipulators. In Intelligent Robots and Systems (IROS), 2011 IEEE/RSJ International Conference on, pages 1036-1041. IEEE, 2011.

[20] L. Sentis. Synthesis and Control of Whole-Body Behaviors in Humanoid Systems. PhD thesis, Stanford University, Stanford, USA, 2007.

[21] L. Sentis and O. Khatib. Synthesis of whole-body behaviors through hierarchical control of behavioral primitives. International Journal of Humanoid Robotics, 2(4):505-518, December 2005.

[22] R. Tellez, F. Ferro, S. Garcia, E. Gomez, E. Jorge, D. Mora, D. Pinyol, J. Oliver, O. Torres, J. Velazquez, and D. Faconti. Reem-b: An autonomous lightweight human-size humanoid robot. In Humanoid Robots, 2008. Humanoids 2008. 8th IEEE-RAS International Conference on, pages $462-468$, dec. 2008.

[23] N.D. Vuong, MH Ang, T.M. Lim, and S.Y. Lim. An analysis of the operational space control of robots. In Robotics and Automation (ICRA), 2010 IEEE International Conference on, pages 4163-4168. IEEE, 2010. 